\title{
Incorporating Quantum Number Characteristics in the Pictorial Representations of the Atomic Structure: Consistency Issues and Students' Relevant Profiles
}

\author{
Nikolaos Zarkadis, George Papageorgiou*, Angelos Markos \\ Department of Primary Education, Democritus University of Thrace, Alexandroupolis, Greece \\ *Corresponding Author: gpapageo@eled.duth.gr
}

\section{ABSTRACT}

The main purpose of this study was to investigate the consistency of pictorial representations of the atomic structure drawn by students on the basis of particular quantum numbers' characteristics. Participants were 192 students of the $12^{\text {th }}$ grade of secondary Greek schools. This study's instrument, a paper-and-pencil assessment tool, included four open-ended tasks. In each one of the four tasks, students were asked to draw representations of the atomic structure under two specific conditions concerning particular quantum numbers' values. Results indicated the existence of a consistency between pictorial representations, providing indications that this kind of representation could be used as a consistent tool in order to study students' understanding of the contribution of quantum number characteristics in the configuration of the atomic structure. Furthermore Latent Class Analysis revealed distinct student profiles indicating how this consistency is distributed across the sample and emerging student classes that have problems in conceptualizing the characteristics of the first and fourth quantum numbers. Implications for science education are discussed.

KEY WORDS: Atomic structure; consistency; latent class analysis; pictorial representations, quantum numbers

\section{INTRODUCTION}

" uantum numbers" understanding is one of the major factors associated with a scientifically accepted consideration of the atomic structure within the quantum context. However, such an understanding is quite difficult, since quantum numbers are introduced as particular numbers that derive during the solution of the "Schrödinger equation" and can determine characteristics of the orbitals such size, shape, and orientation. Thus, the abstract, symbolic, and sophisticated character of quantum numbers have caused a number of student conceptual difficulties and inconsistencies concerning electronic configuration problems and the understanding of the atomic structure, in general (Ardac, 2002; Papaphotis and Tsaparlis 2008; Park and Light, 2009; Sunyono et al., 2016; Taber, 2002; Temel and Özcan, 2018; Tsaparlis and Papahotis, 2009).

As a number of researchers suggest, students have difficulties in interpreting the values of quantum number when determining the quantum status of an electron or writing the electron configuration. Thus, although the first quantum number $n$ (the "principal quantum number") specifies the size of an orbital and the second one $l$ (the "angular momentum quantum number") specifies its shape (s, p, d, f), students often fail to connect their values (e.g., 1, 2, 3... of $n$ ) or the corresponding symbols (s, p, d...of $l$ ) with particular size and shape. Similarly, although the third quantum number $m_{l}$ (the "magnetic quantum number") specifies the orientation of an orbital in space (in the presence of an external magnetic field), students often use self-generated rules connecting its values $(\ldots-2,-1,0$, $1,2 \ldots)$ with different energy levels, considering that a higher $m_{l}$ value corresponds to a higher energy level (Ardac, 2002; Papaphotis and Tsaparlis, 2008; Sunyono et al., 2016; Taber, 2002; Temel and Özcan, 2018).

As a result, students' difficulties in the conceptualization of quantum numbers are also associated with problems in pictorial representations of the corresponding atomic structure. Based on the relevant literature, these problems appear to be connected to a student's trend to approach quantum numbers through a deterministic way. Thus, in many cases, deterministic or hybrid student representations overshadowed the quantum ones, conflating characteristics of different models and appearing many characteristics of the Bohr's model. For instance, students are often influenced by the Bohr's model ideas and use the concepts of shells, orbits, and orbitals interchangeably or as synonyms (Allred and Bretz, 2019; Zarkadis et al., 2017; Dangur et al., 2014; Nakiboglu, 2003; Özcan, 2013; Papaphotis and Tsaparlis, 2008; Sunyono et al., 2016; Taber, 2002, 2005; Temel and Özcan, 2018; Tsaparlis and Papaphotis, 2009).

Despite the determinative role of quantum numbers ideas in the pictorial representation of the atomic structure, so far, there is no systematic study providing evidence for the consistency of such a kind of representation as for its ability to depict realistically what students have in their minds for the quantum numbers. Could we consider students' pictorial representations 
as a reliable consistent tool for this purpose, and for how many students could this holds true (i.e., what is the student profile responding to such a consistency)? The present study was targeted to seek answers to such questions.

\section{Students' Representations of the Atomic Structure and their Consistency}

Searching the relevant literature, one can find quite a lot of categorizations of students' representations of the atomic structure. One the most frequently found categorizes them into the following five categories (Papageorgiou et al., 2016; Park and Light, 2009; Zarkadis et al., 2017):

- The "atom-cell model" category: The atom is similar to a cell, and it considers being a living organism.

- The "particle model" category: The atom is a particle without further submicroscopic characteristics.

- The "nuclear model" category: The atom is an entity with its components at the submicroscopic level.

- The "Bohr's model" category: The atom contains particular paths of electrons (with or without reference to energy quantization).

- The "quantum model" category: It recognizes the probabilistic view of the atom.

However, among the above five categories, recent studies pay particular attention to the last category during the latest years, focusing on whether or not students can move within the quantum/probabilistic context (Dangur et al., 2014; Papageorgiou et al., 2016; Zarkadis et al., 2017; Zarkadis and Papageorgiou, 2020). Thus, generally, students' ideas can be characterized as quantum, where the probabilistic context has been established, or deterministic, where most of the students express deterministic/mechanistic ideas in accordance with "Bohr's model." However, there are also cases, where characteristics of the quantum and the deterministic categories coexist and thus, such cases are seen to form a separate category, the hybrid category. Characteristics example of this case include considerations of orbitals as alternative shapes of orbits, drawings presenting electron clouds moving on specific orbits, or confusing the concept of orbit with that of the probability (Kiray, 2016; Park and Light, 2009; Tsaparlis and Papaphotis, 2009).

The existence of the hybrid category indicates, in fact, an inconsistency in adopting characteristics solely of a particular model (i.e., quantum or deterministic) and contributes to the aspect that knowledge is fragmented (diSessa, 1993; 2014; diSessa et al., 2004). According to this aspect, "knowledge elements" have been formed consciously or unconsciously in students' mind over time, during learning or cognitive processes, and pre-exist when further educational processes take place (i.e., when particular topics are being studied). These elements are considered to be flexible pieces of knowledge that can be activated according to the demands of the context of the topic under study. They can be also evolved, modified, and coordinated with other pieces of knowledge forming bigger cognitive structures facilitating a particular concept or phenomenon. Consequently, this aspect of fragmented knowledge can support the formation of such hybrid models by the association of knowledge elements coming from both the quantum and the deterministic models of the atomic structure. This kind of inconsistency has been characterized as an inconsistency "within" the models (Zarkadis et al., 2017).

However, there are cases where, the context of a task or a situation can lead students to the adoption of a particular model of the atomic structure, whereas when the context changes, then students are led to the adoption of a different model (Papageorgiou et al., 2016). This contradicts the aspect that students construct mental models that are coherent and stable independently of the topic is being studied (Vosniadou and Brewer, 1992, 1994). These coherent mental models appear to be consistent throughout a variety of tasks, phenomena, or situations and for this reason, they are characterized as "theory-like." On the contrary, the aspect of "fragmented knowledge" supports the possibility for the students to form different models depending on the particular topic under study, combining different "knowledge elements" each time. Thus, this could be considered as another kind of "inconsistency," which has been characterized as an inconsistency "between" the models (Zarkadis et al., 2017).

\section{Greek Instructional Context for the Quantum Numbers}

According to the Greek National Science Curriculum, students of the secondary education (Grades 7-12) are introduced to the concept of the atom and its characteristics in the $8^{\text {th }}$ grade, whereas during the $10^{\text {th }}$ grade they are taught the concept of electron configuration, including the "principal quantum number," the energy quantization and the concept of shell, in the context of the Bohr's model. In the $12^{\text {th }}$ grade, students have to follow one of three directions, i.e., "science and mathematics," "humanities," and "economy and information." Quantum numbers are taught in the "science and mathematics" direction for three 1-h lessons per week. The relevant part of the curriculum comprises the teaching of the quantum model and related concepts, such as the wave-particle duality, the uncertainty principle, the wavefunction, the atomic orbital, the electron cloud and its density, the quantum numbers, their value and what they represent, as well as, graphical representations of the hydrogenic atomic orbital types and shapes in terms of their electron densities.

\section{Rationale of the Study and Research Questions}

The present study was a part of a wider project aiming to access student understanding of quantum numbers and its effects on both the verbal and pictorial representations of the atomic structure (see also Zarkadis et al., 2021). Participants are students of the $12^{\text {th }}$ grade, who have been taught the quantum model, but they were also aware of the Bohr's atomic model, as they had received relevant lessons in the $8^{\text {th }}$ and $10^{\text {th }}$ grades. As a result, it is possible that students have conceptualized quantum numbers' ideas within the corresponding quantum context, or to have been influenced by the Bohr's deterministic ideas to a certain degree. Thus, 
corresponding students' pictorial representations of the atomic structure could incorporate quantum numbers' ideas within quantum, deterministic or hybrid context, presenting also differences/inconsistencies depending on the particular characteristics/values of these quantum numbers. In this context, an investigation of the consistency between different pictorial representations of the atomic structure takes place in the present study, when particular quantum numbers' characteristics are given. In particular, the following research questions are under investigation:

1. How do students represent pictorially the atomic structure when different quantum numbers' characteristics/values are given? Is there any consistency between these representations?

2. Which distinct student profiles or typologies emerge in relation to their pictorial representations of the atomic structure?

\section{METHODOLOGY}

\section{Sample}

Participants were 192 students of the $12^{\text {th }}$ grade of secondary schools from Northern Greece, 105 (54.7\%) male, and 87 (45.3\%) female. All participants were following the Greek National Science Curriculum for the "science and math" direction (Greek Pedagogical Institute, 2003) using the same textbook. Furthermore, they were from mixed socio-economic backgrounds, attending classes of regular public schools. Prior to the study, informed consent was obtained from the teachers and the heads of the participating schools, as well as from the students who participated in the study, being aware of the purpose of the study. Participation was anonymous, voluntary and confidential, without personal benefit, whereas the study was approved by the Department of Primary Education of the Democritus University of Thrace, Greece, in accordance with guidelines provided by the Institution's Ethics Committee.

\section{Instrument}

The research tool was an anonymous paper-and-pencil test including four open-ended tasks (Table 1), which was developed for the needs of the present study, taking into account relevant literature (Akaygun, 2016; Dangur et al., 2014). Its completion took place during the last semester of the school year and lasted $45 \mathrm{~min}$.

In each one of the four tasks, students were asked to draw representations of the atomic structure under two specific conditions concerning quantum number characteristics/values, naming any component of their drawing, and explaining how these different conditions lead to different corresponding atomic representations. Before the main study, the instrument was tested during a pilot study, which was conducted with 74 students. No problems were noted and thus, no changes were made to the final tool.

\section{Data Analysis}

Student pictorial representations were evaluated on the basis of relevant coding schemes already presented in the literature (Akaygun, 2016; Dangur et al., 2014; Tang et al., 2019), focusing on the components: (a) electrons or electron clouds (as for their location, alignment, scale, shape, size, orientation, and spin orientation) and (b) orbitals (as for their location, alignment, scale, shape, size, and orientation). The evaluation of each one of these components was based on its correctness and completeness, taking also into account that students' drawings might have been influenced by the corresponding images of the textbooks, which are designed in the context of the school level of the scientific view (Koulaidis and Tsatsaroni, 1996; Tsaparlis and Papaphotis, 2002). Note that, in the term completeness, also the complexity is included (see, for instance, Dangur et al., 2014), referring to whether the drawing is rich and detailed as for the number of items (e.g., orbital shapes, axis, angles, or dots) included in each drawing.

The coding process was conducted by the first and second authors and any discrepancies between them were discussed and resolved until consensus was reached. Table 2 shows the final coding scheme, including categories quantum, hybrid or deterministic with subcategories. Some characteristic examples are presented in Figure 1.

\section{Statistical Analysis}

The construct validity of the instrument was examined in the context of Confirmatory Factor Analysis (CFA). Due to the ordinal nature of the test items, the analysis was performed using the WLSMV estimator, implemented in Mplus 8.4 (Muthén and Muthén, 2017). Model fit was evaluated using the following indices: Comparative fit index (CFI), Tucker-

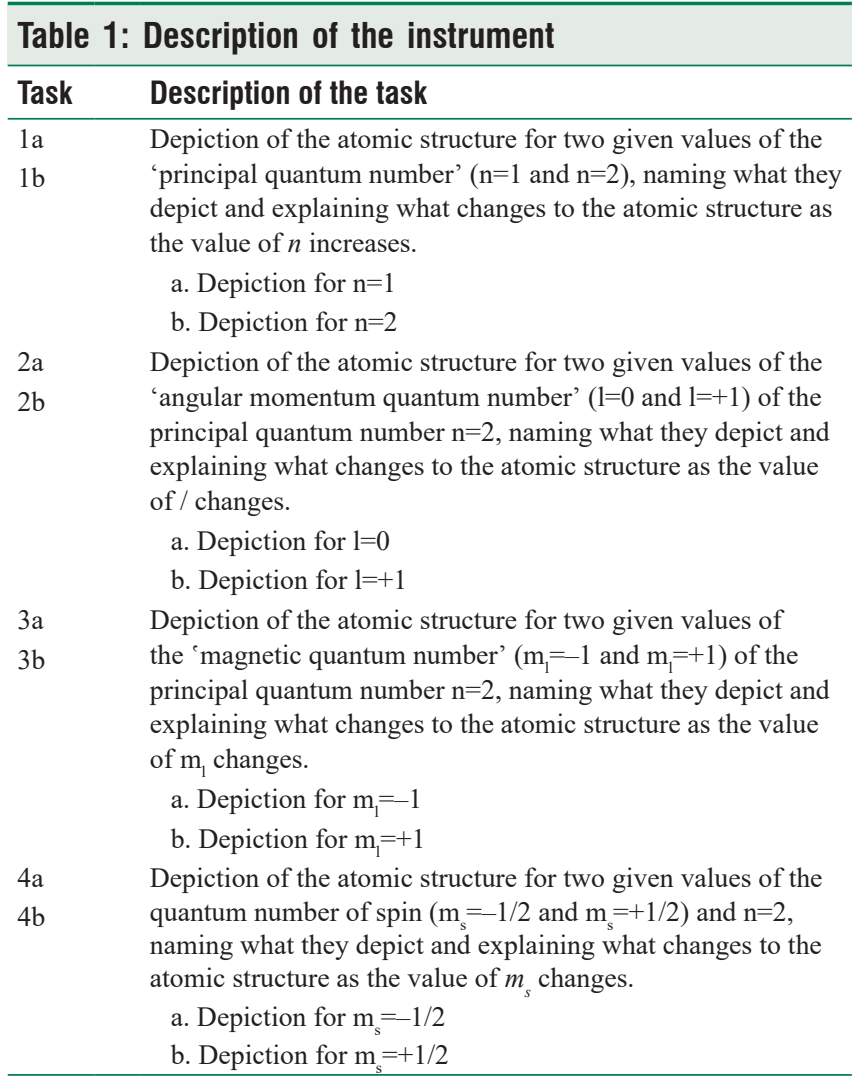




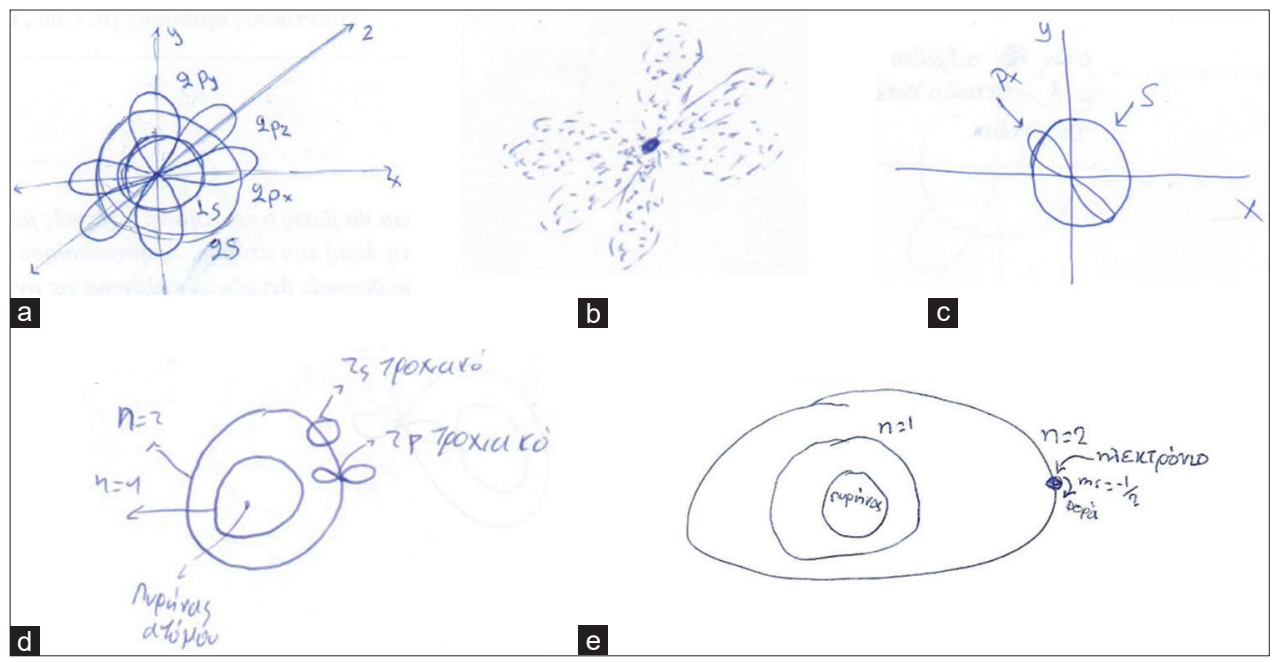

Figure 1: Some characteristic examples of Quantum model Q3 (a), Quantum model Q2 (b), Quantum model Q1 (c), Hybrid model H (d), and Deterministic model D (e)

\begin{tabular}{|c|c|c|}
\hline Model & Category & Description \\
\hline Quantum & Q3 & $\begin{array}{l}\text { All quantum numbers characteristics are } \\
\text { completely and correctly depicted with } \\
\text { high complexity within the quantum } \\
\text { model. }\end{array}$ \\
\hline Quantum & Q2 & $\begin{array}{l}\text { Some quantum numbers characteristics } \\
\text { are completely and correctly depicted } \\
\text { within the quantum model (correct } \\
\text { but incomplete representation - low } \\
\text { complexity). }\end{array}$ \\
\hline Quantum & Q1 & $\begin{array}{l}\text { Incorrect (complete or not) } \\
\text { representation within the quantum } \\
\text { model (it includes incorrect quantum } \\
\text { numbers characteristics). }\end{array}$ \\
\hline Hybrid & $\mathrm{H}$ & $\begin{array}{l}\text { Correct or incorrect (complete or not) } \\
\text { representation with quantum numbers } \\
\text { characteristics being depicted in a mixed } \\
\text { way referring to both deterministic and } \\
\text { quantum models. }\end{array}$ \\
\hline Deterministic & $\mathrm{D}$ & $\begin{array}{l}\text { Correct or incorrect (complete or not) } \\
\text { representation with quantum numbers } \\
\text { characteristics being depicted within a } \\
\text { deterministic context. }\end{array}$ \\
\hline None & $\mathrm{X}$ & Unclear drawing or no response. \\
\hline
\end{tabular}

Lewis index (TLI), root mean square error of approximation (RMSEA), and 90\% confidence interval (CI) of RMSEA. CFI and TLI values $\geq 0.95$ and RMSEA values $\leq 0.08$, were considered as good indicators of model fit $(\mathrm{Hu}$ and Bentler, 1999). CFA was conducted using the $R$ package lavaan (Rosseel, 2012). Cronbach's alpha and McDonald's omega were calculated as internal consistency indicators, using the $\mathrm{R}$ package sem Tools (Jorgensen et al., 2021).

The consistency between pictorial representations for each task (1a vs. 1b, 2a vs $2 b$, etc.) was statistically assessed in terms of the symmetry of the corresponding contingency sub-tables (Table 3 in the Results section). The McNemarBowker test was used in order to evaluate the symmetry of each contingency sub-table, or a Monte Carlo multinomial exact test with 1,000 trials, alternatively, in cases where the assumptions of McNemar-Bowker test were not met. Both tests resulted in a two-sided $p$-value, testing the null hypothesis that a contingency sub-table is symmetric. The rejection of the null hypothesis indicates the existence of significant transitions of student representation from a category to another in the contingency sub-table. Post-hoc McNemar tests with Bonferroni adjusted alpha levels were also performed in order to further determine any statistically significant transition. The tests were conducted with the functions mcnemar.test and nominalSymmetryTest of the $\mathrm{R}$ package rcompanion (Mangiafico, 2017).

To identify classes of students with similar profiles across the set of tasks concerning their knowledge and representational competence for the atomic structure based on the quantum numbers, we used latent class analysis (LCA). LCA is a model-based approach that uses multiple indicators to estimate unobservable distinct subgroups defined as latent classes in multivariate categorical data (for similar applications see Harlow et al., 2011). The latent classes are identified by means of statistical criteria and they are characterized by a number of conditional probabilities. These are the probabilities of providing a certain response to a given task, given that a student belongs to the specific latent class. To select the optimal number of latent classes, we used the Bayesian information criterion (BIC) combined with conceptual appeal (interpretability) of the resulting solution (Collins and Lanza, 2010). For this reason, profile size was also considered when determining the optimal number of profiles: models that produced profiles representing less than $5 \%$ of the total sample were not further examined. In addition, to assess the precision with which the participants were classified into latent profiles, the entropy value was considered. A higher value of entropy (i.e., closer to 1 ) indicates better latent profile separation (Berlin et al., 2014). To test 
the validity of the local independence assumption, bivariate residuals (BVRs) were analyzed. Values of BVRs greater than 3.84 identify correlations between pairs of indicators that have not been explained by the model at $\alpha=.05$ LCA. LCA was carried out using Latent Gold version 5.1 (Vermunt and Magidson, 2016).

\section{RESULTS}

\section{Descriptive Analysis}

Table 4 shows that a large percentage of students identified a deterministic model (D) in tasks $1 \mathrm{a}$ and $1 \mathrm{~b}$ (47\% and $43 \%$, respectively), compared to the remaining tasks ( $2 \mathrm{a}$ to $4 \mathrm{~b}$ ), where student percentages in model D range from $5 \%$ to $25 \%$. Moreover, the distribution of student percentages in tasks $3 \mathrm{a}$ and $3 \mathrm{~b}$, as well as in tasks $4 \mathrm{a}$ and $4 \mathrm{~b}$, seems to be similar comparing cases $a$ and $b$ to each other, whereas it could be said that tasks $2 \mathrm{a}$ and $2 \mathrm{~b}$ have also some similarities to each other, in the sense that the vast majority of students $(>80 \%)$ holds a quantum model, whether it is correct (Q2 or Q3) or not (Q1). For instance, for task 2a $59 \%$ of students provided a response that was classified as correct (Q2 or Q3) and 22\% as incorrect (Q1). The distribution of student percentages in tasks $4 \mathrm{a}$ and $4 \mathrm{~b}$ are somewhat different from the others, where $25 \%$ of the students appear to hold a deterministic model.

\section{Construct Validity and Reliability}

Two unidimensional CFAs demonstrated a good fit to the data for both conditions concerning quantum number characteristics/values, i.e., for items $1 \mathrm{a}, 2 \mathrm{a}, 3 \mathrm{a}$ and $4 \mathrm{a}\left(\chi^{2}(2)\right.$ $=4.026, p=0.134, \mathrm{CFI}=0.97, \mathrm{TLI}=0.91, \mathrm{RMSEA}=0.047$ [0.045-0.049], SRMR $=0.047)$ and items $1 b, 2 b, 3 b$ and $4 b$ $\left(\chi^{2}(2)=1.391, p=0.134, \mathrm{CFI}=0.98, \mathrm{TLI}=0.92\right.$, RMSEA $=0.028[0.025-0.032]$, SRMR $=0.031)$. McDonald's omega and Cronbach's alpha indicated that the two groups of items exhibited satisfactory internal consistency $(\omega=$ $0.65, \alpha=0.67$ for items 1 a to $4 \mathrm{a}$ and $\omega=0.66, \alpha=0.69$ for items $1 b$ to $4 b)$.

Table 3: Crosstabulation of student pictorial representations (each sub-table corresponds to a task)-number of students in each category

\begin{tabular}{|c|c|c|c|c|c|c|c|c|c|c|c|c|c|c|c|c|c|c|}
\hline \multirow[t]{2}{*}{ Task 1a } & \multicolumn{7}{|c|}{ Task 1b } & \multirow[t]{2}{*}{ Task 2a } & \multicolumn{10}{|c|}{ Task 2b } \\
\hline & & D & H & Q1 & Q2 & Q3 & Total & & $X$ & & D & & H & & & Q2 & Q3 & Total \\
\hline & & & & & & & & $\mathrm{X}$ & 5 & & 0 & & 0 & & & 0 & 0 & 5 \\
\hline D & & 82 & 2 & 4 & 2 & 1 & 91 & $\mathrm{D}$ & 0 & & 9 & & 0 & & & 2 & 0 & 14 \\
\hline $\mathrm{H}$ & & 1 & 11 & 0 & 0 & 0 & 12 & $\mathrm{H}$ & 0 & & 0 & & 14 & & & 4 & 0 & 18 \\
\hline Q1 & & 0 & 0 & 0 & 2 & 0 & 2 & Q1 & 0 & & 0 & & 0 & & & 20 & 2 & 42 \\
\hline Q2 & & 0 & 0 & 4 & 6 & 0 & 10 & Q2 & 0 & & 0 & & 0 & & & 26 & 3 & 40 \\
\hline Q3 & & 0 & 0 & 3 & 51 & 23 & 77 & Q3 & 0 & & 0 & & 0 & & & 37 & 32 & 73 \\
\hline Total & & 83 & 13 & 11 & 61 & 24 & 192 & Total & 5 & & 9 & & 14 & & & 89 & 37 & 192 \\
\hline \multirow[t]{2}{*}{ Task 3a } & \multicolumn{7}{|c|}{ Task 3b } & Task 4a & \multicolumn{10}{|c|}{ Task 4b } \\
\hline & $X$ & D & $\mathrm{H}$ & Q1 & Q2 & Q3 & Total & & $X$ & D & & $\mathrm{H}$ & & Q1 & Q2 & & & Total \\
\hline $\mathrm{X}$ & 11 & 0 & 0 & 0 & 0 & 0 & 11 & $X$ & 16 & 0 & & 0 & & 0 & 0 & & & 16 \\
\hline $\mathrm{D}$ & 0 & 9 & 0 & 0 & 0 & 0 & 9 & $\mathrm{D}$ & 0 & 47 & & 0 & & 0 & 0 & & & 47 \\
\hline $\mathrm{H}$ & 0 & 0 & 9 & 0 & 0 & 0 & 9 & $\mathrm{H}$ & 1 & 0 & & 12 & & 0 & 0 & & & 13 \\
\hline Q1 & 3 & 0 & 0 & 35 & 3 & 0 & 41 & Q1 & 0 & 0 & & 0 & & 36 & 0 & & & 36 \\
\hline Q2 & 2 & 0 & 0 & 8 & 93 & 0 & 103 & Q2 & 1 & 0 & & 0 & & 0 & 70 & & & 71 \\
\hline Q3 & 0 & 0 & 0 & 2 & 2 & 15 & 19 & Q3 & 0 & 0 & & 0 & & 0 & 0 & & & 9 \\
\hline Total & 16 & 9 & 9 & 45 & 98 & 15 & 192 & Total & 18 & 47 & & 12 & & 36 & 70 & & & 192 \\
\hline
\end{tabular}

Significant differences are shown in bold and italics

Table 4: Students' numbers and percentages on pictorial representations (tasks $1 \mathrm{a}$ to $4 \mathrm{~b}$ )

\begin{tabular}{|c|c|c|c|c|c|c|c|c|c|c|c|c|c|c|c|c|}
\hline \multirow[t]{3}{*}{ Category } & \multicolumn{16}{|c|}{ Tasks } \\
\hline & \multicolumn{2}{|c|}{$1 a$} & \multicolumn{2}{|c|}{$1 b$} & \multicolumn{2}{|c|}{$2 a$} & \multicolumn{2}{|c|}{$2 b$} & \multicolumn{2}{|c|}{$3 a$} & \multicolumn{2}{|c|}{$3 b$} & \multicolumn{2}{|c|}{$4 a$} & \multicolumn{2}{|c|}{$4 b$} \\
\hline & $f$ & $\%$ & $f$ & $\%$ & $f$ & $\%$ & $f$ & $\%$ & $f$ & $\%$ & $f$ & $\%$ & $t$ & $\%$ & $f$ & $\%$ \\
\hline $\mathrm{X}$ & 0 & 0 & 0 & 0 & 5 & 3 & 5 & 3 & 11 & 6 & 16 & 8 & 16 & 8 & 18 & 9 \\
\hline $\mathrm{D}$ & 91 & 47 & 83 & 43 & 14 & 7 & 9 & 5 & 9 & 5 & 9 & 5 & 47 & 24 & 47 & 25 \\
\hline $\mathrm{H}$ & 12 & 6 & 13 & 7 & 18 & 9 & 14 & 7 & 9 & 5 & 9 & 5 & 13 & 7 & 12 & 6 \\
\hline Q1 & 2 & 1 & 11 & 6 & 42 & 22 & 38 & 20 & 41 & 21 & 45 & 23 & 36 & 19 & 36 & 19 \\
\hline Q2 & 10 & 5 & 61 & 32 & 40 & 21 & 89 & 46 & 103 & 54 & 98 & 51 & 71 & 37 & 70 & 37 \\
\hline Q3 & 77 & 40 & 24 & 12 & 73 & 38 & 37 & 19 & 19 & 10 & 15 & 8 & 9 & 5 & 9 & 5 \\
\hline
\end{tabular}

(X) no model, (D) deterministic model, (H) hybrid model, (Q1) quantum model (incorrect), (Q2) quantum model (correct but incomplete), and (Q3) quantum model (correct and complete) 


\section{Consistency within Pictorial Representations}

Monte Carlo multinomial exact tests of symmetry showed only a few significant transitions from task $1 \mathrm{a}$ to task $1 \mathrm{~b}(\mathrm{p}<0.001)$, as well as from task $2 a$ to task $2 b(p<0.001)$. Significant transitions were observed in student responses from Q3 to Q2 for pairs task 1a - task 1b and task 2a - task 2b (Table 3). However, note that since Q2 and Q3 are two adjacent categories indicating sufficient knowledge, we can assume consistency in the corresponding representations. Moreover, consistency was confirmed for representations pairs task $3 \mathrm{a}$ - task $3 \mathrm{~b}$ and task $4 \mathrm{a}$ - task $4 \mathrm{~b}$ (Table 3 ), as indicated by non-significant two-sided $\mathrm{p}$ values.

\section{Student Profiles in Knowledge and Representational Competence}

LCA was conducted to identify classes of students characterized by particular profiles of representational competence across all tasks. Student responses in all eight tasks of pictorial representations entered the model as the observed variables. Models with a range of one through eight latent classes were estimated with 1,000 random sets of starting values, showing that the model with five classes had the lowest BIC value. This model had an entropy $\mathrm{R}^{2}$ value of 0.94 and a classification error of $0.6 \%$, indicating that the five classes differentiated between the students. No BVR in the five-profile model exceeded the value of 3.84. These values do not indicate a violation of the assumption of local independence.

Figure 2 shows the estimated probability of providing a scientifically sufficient response to each task, given that a student belongs to a specific cluster. Cluster 1, with a prevalence of $35 \%$, is characterized by a high likelihood of providing a scientifically sufficient representation to all tasks. This is clearly the largest cluster. Cluster $2(11 \%)$ is characterized by a high likelihood of providing a scientifically sufficient representation to all tasks but $4 \mathrm{a}$ and $4 \mathrm{~b}$. Cluster
$3(16 \%)$ is characterized by a low likelihood of providing a scientifically sufficient representation to all tasks. Students in Cluster $4(17 \%)$ are characterized by a high likelihood of providing a sufficient representation to tasks $2 \mathrm{a}, 2 \mathrm{~b}, 3 \mathrm{a}$, and $3 \mathrm{~b}$. Last, cluster $5(21 \%)$ is composed of students characterized by a high likelihood of providing a sufficient representation to all tasks but $1 \mathrm{a}$ and $1 \mathrm{~b}$.

\section{DISCUSSION AND CONCLUSIONS}

According to the findings of the study, Bohr's model seems to significantly affect pictorial representations of the first and the fourth quantum numbers (Table 4). Deterministic model (D) appears to reach high percentages in both tasks 1a and $1 \mathrm{~b}$ (Table 4) with students failing to connect the "size" with a representation of orbitals in a 3D space, representing very often shells around the nucleus, in accordance with relevant literature (Dangur et al., 2014; Papageorgiou et al., 2016; Park and Light, 2009). Deterministic approaches are also quite often in both tasks $4 \mathrm{a}$ and $4 \mathrm{~b}$ (e.g., Figure 1e) highlighting students' difficulties in understanding spin as a quantum idea (see also for instance, Özcan, 2013; Taber, 2002; 2005; Temel and Özcan, 2018).

As for the consistency/inconsistency within students' pictorial representations, it is rather apparent that changes in the value of any quantum number do not significantly differentiate their representations (Table 3), indicating significant consistence. The only significant transitions were recorded when category Q3 of task 1a fell to Q2 of task 1b, as well as when category Q3 of task 2a fell to Q2 of task 2b. However, these cases could be characterized as justified, since cases $b$ were always more sophisticated compared to cases a. Thus, in general, pictorial representations could be considered as consistent tools providing valuable information about students' view of the atomic structure.

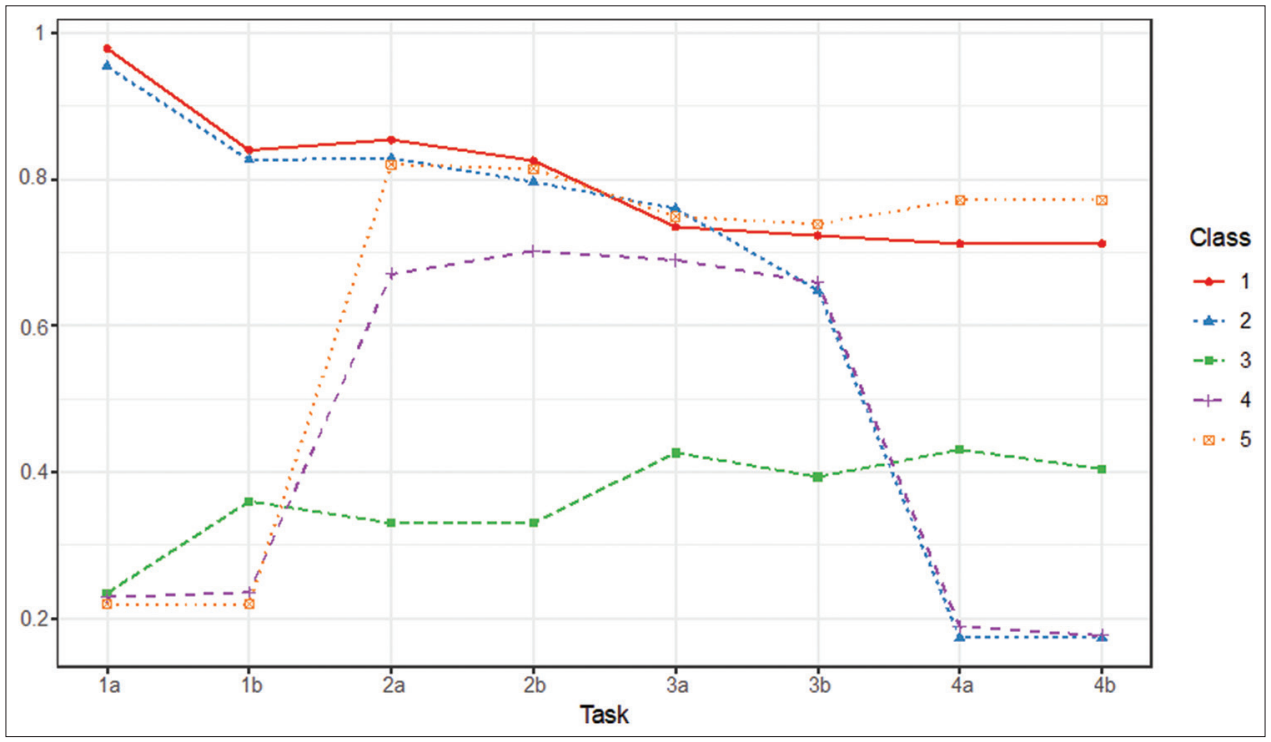

Figure 2: Five student profiles resulting from LCA with the estimated probability (vertical axis) of providing a scientifically sufficient representation to each task (horizontal axis) 
The distribution of the degree of this consistency of pictorial representations among students is provided by studying their profiles. All five profiles provide evidence for the consistency within students' pictorial representations, as the estimated probabilities for a scientifically sufficient response to cases $a$ and $b$ in each one of the tasks are similar in all clusters (Figure 2). This is more obvious in clusters 2, 4, and 5, where significant differences are seen across the tasks. Thus, it is characteristic for cluster 2 that, the low likelihood of providing a scientifically sufficient response to task $4 \mathrm{a}$ is accompanied by an also low likelihood in the case of task $4 \mathrm{~b}$ (whereas all the other cases of the tasks $1 \mathrm{a}$ to $3 \mathrm{~b}$ are characterized by a high likelihood). Similarly, in cluster 5, the low likelihood of providing a scientifically sufficient response to task 1a is accompanied by an also low likelihood in the case of task $1 b$ (whereas all the other cases of the tasks $2 a$ to $4 b$ are characterized by a high likelihood). In addition, in cluster 4 , the same low likelihood holds true for tasks 1a, 1b, 4a, and $4 \mathrm{~b}$, when all the other cases of the rest tasks are characterized by a high likelihood. Thus, it is more apparent that students' relevant conceptual problems concern mainly the first and fourth quantum numbers. However, the fact that pictorial representations appear to be quite reliable tools due to their consistency, could help teachers in order to make more understandable the meaning of the corresponding verbal descriptions concerning the quantum characteristics of these two numbers. As for the other two student profiles, clusters 1 and 3 represent the expected existence of students with high competence in all tasks and those with low competence in all tasks, respectively.

\section{Implications for Science Education}

Conclusions give the possibility of a re-evaluation of the way in which, quantum numbers characteristics can be incorporated in the pictorial representations of the atomic structure in terms of teaching and assessment methodology.

Since the influence of the Bohr's context appears to be stronger in the cases of the fourth and especially of the first quantum number for a significant part of the students (profiles 2, 4, and 5), teachers and textbooks should pay attention to the probabilistic view of the orbital and electron cloud concepts as well as of the angular momentum. Although it is quite difficult to leave one particular context in order to adopt another, an appropriate teaching methodology could probably combine verbal approaches together with the pictorial ones in order to lead students to a disengagement of the deterministic interpretations of these quantum numbers meanings and to facilitate them to make their own scientifically acceptable representations.

This combination of verbal and pictorial approaches could also play an important role in the design of an assessment tool for students' understanding of the quantum number meaning. As a number of researchers suggest (Akaygun and Jones, 2014; Dangur et al., 2014; Ehrlén, 2009) these two kinds of approaches have a complementary role and thus, their interrelation can give a more integrated view of what students have in their mind. Pictorial representations have emerged as a consistent tool for the students' assessment, but promptings for verbal clarifications about the meaning of these representations (concerning, for instance, the location, alignment, scale, shape, size, and orientation of orbitals and electron clouds) could give the opportunity (a) to students in order to express better the way in which consider quantum numbers meaning and (b) to teachers in order to identify possible problematic aspects of their teaching methodology.

\section{Limitations}

The results of the study have a restricted generalization due to the implementation of the measurements in a limited geographical area and in a relatively small sample size. Also, causal inferences could not be safely drawn due to the crosssectional design of the study, whereas the coding scheme that has been used in order to analyze the students' pictorial representations is subject of the researchers' perceptions. The lack of possibilities to interview some of the students in order to clarify details of their representations is also a limitation of the study.

\section{REFERENCES}

Akaygun, S. (2016). Is the oxygen atom static or dynamic? The effect of generating animations on students'. Mental models of atomic structure. Chemistry Education Research and Practice, 17(4), 788-807.

Akaygun, S., \& Jones, L.L. (2014). Words or pictures: A comparison of written and pictorial explanations of physical and chemical equilibria. International Journal of Science Education, 36(5), 783-807.

Allred, Z.D.R., \& Bretz, S.L. (2019). University chemistry students' interpretations of multiple representations of the helium atom. Chemistry Education Research and Practice, 20(2), 358-368.

Ardac, D. (2002). Solving quantum number problems: An examination of novice performance in terms of conceptual base requirements. Journal of Chemical Education, 79(4), 510-513.

Berlin, K.S., Williams, N.A., Parra, G.R. (2014). An introduction to latent variable mixture modeling (Part 1): Overview and cross-sectional latent class and latent profile analyses. Journal of Pediatric Psychology, 39(2), 174-187.

Collins, L.M., \& Lanza, S.T. (2010), Latent Class and Latent Transition Analysis: With Applications in the Social Behavioral, and Health Sciences. New Jersey, United States: Wiley.

Dangur, V., Avargil, S., Peskin, U., \& Dori, Y. J. (2014). Learning quantum chemistry via a visual-conceptual approach: Students' bidirectional textual and visual understanding. Chemistry Education Research and Practice, 15(3), 297-310.

diSessa, A.A. (1993). Toward an epistemology of physics. Cognition and Instruction, 10(2-3), 105-225.

diSessa, A.A. (2014). The construction of causal schemes: Learning mechanisms at the knowledge level. Cognitive Science, 38(5), 795-850.

diSessa, A.A., Gillespie, N., \& Esterly, J. (2004). Coherence versus fragmentation in the development of the concept of force. Cognitive Science, 28(6), 843-900.

Ehrlén, K. (2009). Drawings as representations of children's conceptions. International Journal of Science Education, 31(1), 41-57.

Greek Pedagogical Institute. (2003). National Program of Study for Primary and Secondary Education: Science. Greece: Greek Pedagogical Institute Publications.

Harlow, D.B., Swanson, L.H., Nylund-Gibson, K., \& Truxler, A. (2011). Using latent class analysis to analyze children's responses to the question, What is a day? Science Education, 95(3), 477-496.

Hu, L.T., \& Bentler, P.M. (1999). Cutoff criteria for fit indexes in covariance structure analysis: Conventional criteria versus new alternatives. 
Structural Equation Modelling, 6, 1-55.

Jorgensen, T.D., Pornprasertmanit, S., Schoemann, A.M., Rosseel, Y., Miller, P., Quick, C., \& Ben-Shachar, M.S. (2021). SemTools: Useful Tools for Structural Equation Modeling (R Package Version 0.54). Available from: https://www.cran.r-project.org/web/packages/ semTools/index.html [Last accessed on 2021 June 21].

Kiray, S.A. (2016). The pre-service science teachers' mental models for concept of atoms and learning difficulties. International Journal of Education in Mathematics, Science and Technology, 4(2), 147-162.

Koulaidis, V., \& Tsatsaroni, A. (1996). A pedagogical analysis of science textbooks: How can we proceed? Research in Science Education, 26(1), 55-71.

Mangiafico, S.S. (2017). Rcompanion: Functions to Support Extension Education Program Evaluation. $R$ Package Version 2.4.0. Available from: https://www.cran.rproject.org/web/packages/rcompanion/index. html [Last accessed on 2021 June 21].

Muthén, L.K., \& Muthén, B.O. (2017). Mplus User's Guide. $8^{\text {th }}$ ed. Sweden: Muthén \& Muthén.

Nakiboglu, C. (2003). Instructional misconceptions of Turkish prospective chemistry teachers about atomic orbitals and hybridization. Chemistry Education Research and Practice, 4(2), 171-188.

Özcan, Ö. (2013). Investigation of mental models of Turkish pre-service physics students for the concept of "spin". Eurasian Journal of Educational Research, 52, 21-36.

Papageorgiou, G., Markos, A., \& Zarkadis, N. (2016). Students' representations of the atomic structure-the effect of some individual differences in particular task contexts. Chemistry Education Research and Practice, 17(1), 209-219.

Papaphotis, G., \& Tsaparlis, G. (2008). Conceptual versus algorithmic learning in high school chemistry: The case of basic quantum chemical concepts. Part 2. Students' common errors, misconceptions and difficulties in understanding. Chemistry Education Research and Practice, 9(4), 332-340.

Park, E.J., \& Light, G. (2009). Identifying atomic structure as a threshold concept: Student mental models and troublesomeness. International Journal of Science Education, 31(2), 233-258.

Rosseel, Y. (2012). Lavaan: An R package for structural equation modeling and more. Version 05-12 (BETA). Journal of Statistical Software, 48(2), $1-36$.
Sunyono, S., Tania, L., \& Saputra, A. (2016). A learning exercise using simple and real-time visualization tool to counter misconceptions about orbitals and quantum numbers. Journal of Baltic Science Education, $15(4), 452-463$.

Taber, K.S. (2002). Conceptualizing quanta: Illuminating the ground state of student understanding of atomic orbitals. Chemistry Education Research and Practice, 3(2), 145-158.

Taber, K.S. (2005). Learning quanta: Barriers to stimulating transitions in student understanding of orbital ideas. Science Education, 89(1), 94116.

Tang, K.S., Won, M., \& Treagust, D. (2019). Analytical framework for student-generated drawings. International Journal of Science Education, 41(16), 2296-2322.

Temel, S., \& Özcan, Ö. (2018). Students' understanding of quantum numbers: A qualitative study. In: SHS Web of Conferences. Vol. 48. Les Ulis, France: EDP Sciences. p. 01002.

Tsaparlis, G., \& Papaphotis, G. (2002). Quantum-chemical concepts: Are they suitable for secondary students? Chemistry Education Research and Practice, 3(2), 129-144.

Tsaparlis, G., \& Papaphotis, G. (2009). High school students' conceptual difficulties and attempts at conceptual change: The case of basic quantum chemical concepts. International Journal of Science Education, 31(7), 895-930.

Vermunt, J.K., \& Magidson, J. (2016). Upgrade Manual for Latent GOLD 5.1. United States: Statistical Innovations.

Vosniadou, S., \& Brewer, W.F. (1992). Mental models of the earth: A study of conceptual change in childhood. Cognitive Psychology, 24, 535-585.

Vosniadou, S., \& Brewer, W.F. (1994). Mental models of the day/night cycle. Cognitive Science, 18, 123-183.

Zarkadis, N., \& Papageorgiou, G. (2020). A fine-grained analysis of students' explanations based on their knowledge of the atomic structure. International Journal of Science Education, 42(7), 1162-1182.

Zarkadis, N., Papageorgiou, G., \& Markos, A. (2021). Understanding quantum numbers: Students' verbal descriptions and pictorial representations of the atomic structure. International Journal of Science Education, 43(13), 2250-2269.

Zarkadis, N., Papageorgiou, G., \& Stamovlasis, D. (2017). Studying the consistency between and within the student mental models for atomic structure. Chemistry Education Research and Practice, 18(4), 893-902.

\section{APPENDIX 1}

The instrument used for the needs of the preset study.

\section{Task 1}

Draw the atomic structure for the values of the 'principal quantum number' $n=1$ and $n=2$. Name what you depict and explain what changes to the atomic structure as the value of $\mathrm{n}$ increases.
a. Depiction for $n=1$
b. Depiction for $n=2$

\section{Task 2}

Draw the atomic structure for the values of the 'angular momentum quantum number' $l=0$ and $l=+1$ of the principal quantum number $n=2$. Name what you depict and explain what changes to the atomic structure as the value of $l$ changes.
a. Depiction for $l=0$
b. Depiction for $l=+1$

\section{Task 3}

Draw the atomic structure for the values of the 'magnetic quantum number' $m_{l}=-1$ and $m_{l}=+1$ of the principal quantum number $n=$ 2. Name what you depict and explain what changes to the atomic structure as the value of $m_{l}$ changes.

a. Depiction for $m_{l}=-1$

b. Depiction for $m_{l}=+1$ 


\section{Task 4}

Draw the atomic structure for the values of the quantum number of spin $m_{s}=-1 / 2$ and $m_{s}=+1 / 2$ when $\mathrm{n}=2$. Name what you depict and explain what changes to the atomic structure as the value of $m_{s}$ changes.

a. Depiction for $m_{s}=-1 / 2$

b. Depiction for $m_{s}=+1 / 2$

\section{APPENDIX 2}

Examples of complete and correct students' representations (Q3 category) for each one of the tasks.
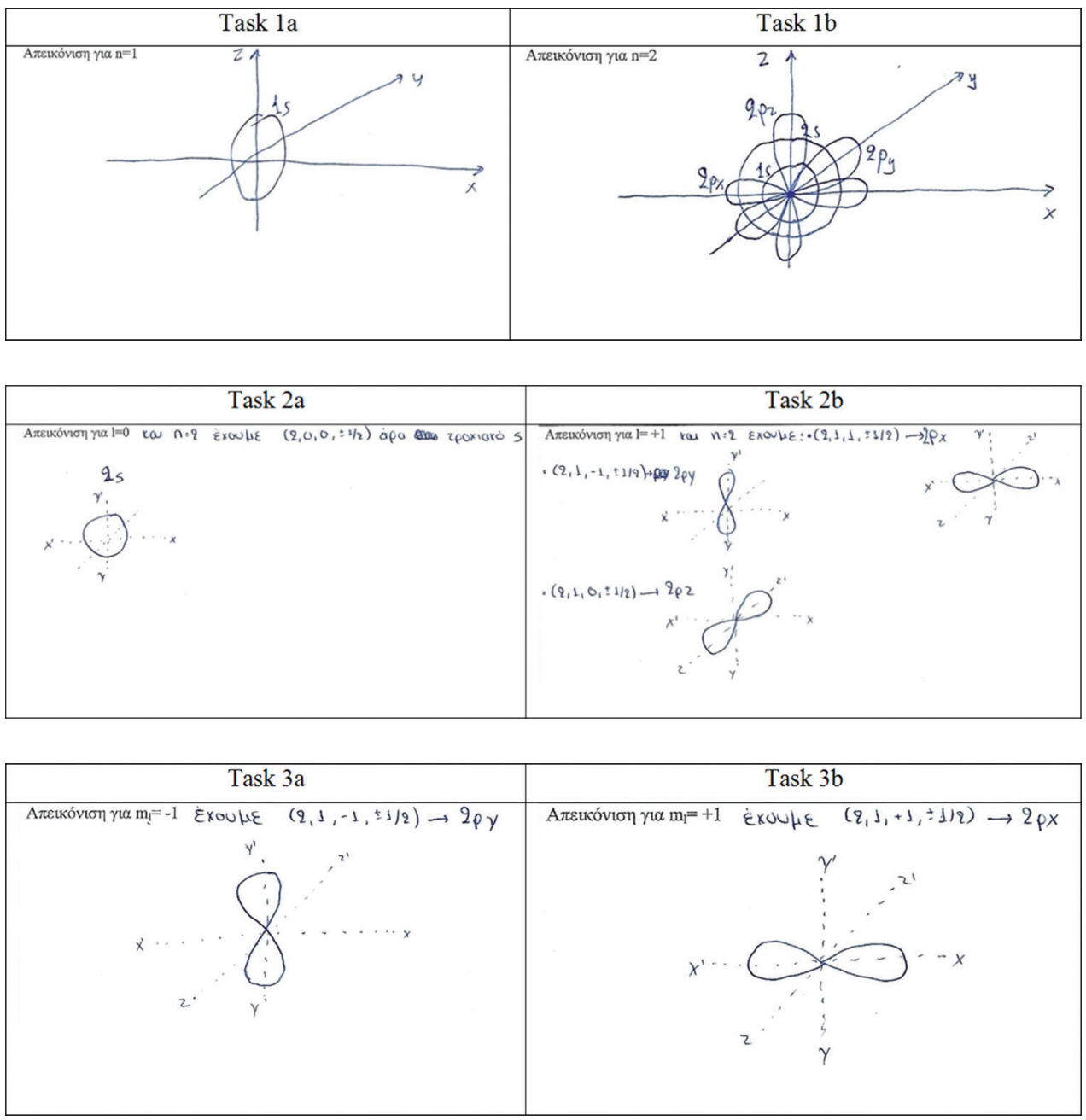

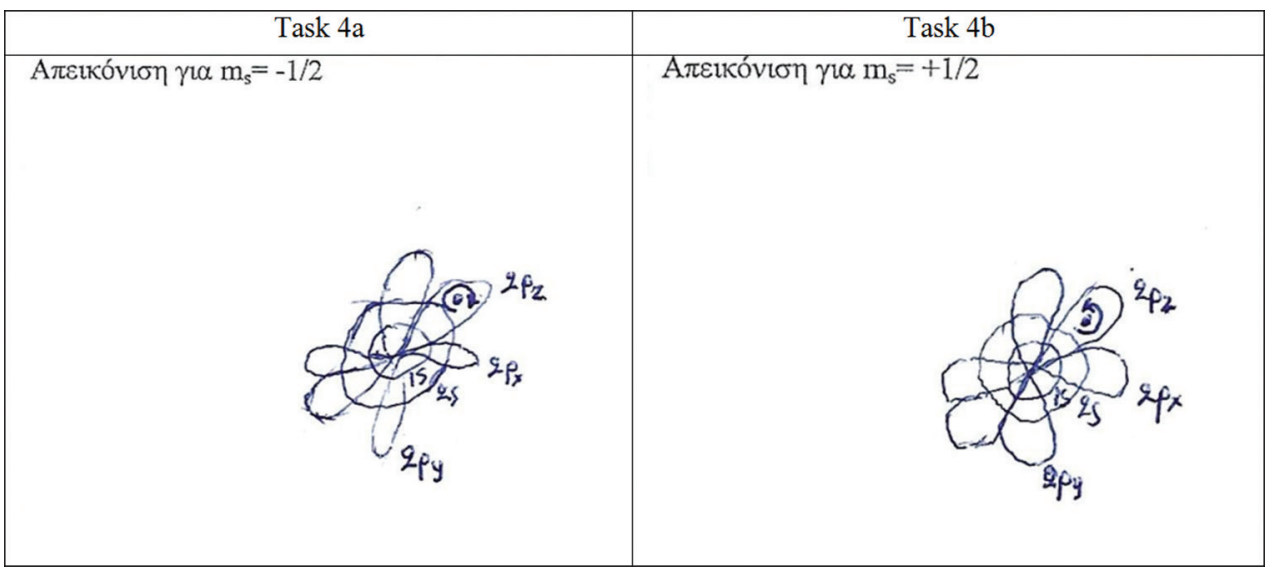

